\title{
THEORY MEETS PRACTICE IN THE DESIGN OF E-SUPPORT FOR JUNIOR REGISTRAR DOCTORS
}

Anne Marie Kanstrup ${ }^{1} \&$ Niels Boye ${ }^{2}$,

'Aalborg University, Aalborg, Denmark \& 2Danish Technological Institute, Aarhus, Denmark

The paper presents a model that operates between theory and practice through the design of a mobile application for learning support, which was developed for junior registrar doctors on a medical ward. The nature of junior doctors' clinical education is learning while producing. While they have a large pool of theoretical knowledge, they must make that knowledge operational for diagnostic and therapeutic considerations and procedures. In order to aid this development, the authors consider models for understanding the process of operating between the work of medicine and medical knowledge. Their design problem led them to a plan in which they identified and synthesized the links between abstract theoretical models and day-to-day practice in medicine, within the constraints of hardware and software in a mobile application, which was designed to support junior registrar doctors in their clinical training. In doing so, a shared language of the design domain within a team of physicians and interaction designers emerged. The paper describes a process where there is no 1:1 relationship between theory and practice and, consequently, suggests the need to understanding the domains of medicine and design in this light.

Keywords: clinical reasoning, handheld computers, interaction design, medical knowledge

Wicked problems (Buchanan, 1996), uncertainty (Mathiassen \& Stage, 1992), and social construction (Floyd, 1992) are characteristics used for describing software design. These characteristics highlight the complexity of the design process where " $A$ " is not necessarily followed by " $B$ " and " $C$ ", and where the truth out there does not exist beforehand but is negotiated or created during the process. In other words, while the theoretical foundation of computer science is in the natural science domain, where most things can be measured and ranked in relation to each other, the process of designing information systems is often closer to human sciences, here understood broadly in relation to decidability. This dualism between natural science in theory and human science in practice is also found within medicine. The foundation of Western medical knowledge is in the natural sciences domain, where most things considered can be measured and ranked in relation to each other. But making the knowledge active to a person or group of persons is considered within the human sciences domain. This shift from natural science to human science makes medical knowledge operational by (1) adding value to the single individual human being's life or (2) creating societal conditions that promote health among a group of people (public health initiatives). In these two major activities medical practice is closer to human sciences.

Lack of awareness concerning this theory/practice predicament in the area of health informatics is often reflected in computer systems for clinical use. These systems attempt to make logical data structures and lower unwanted variation in health provisions by incorporating rigorous models of non-existent "standard patients" and "standard operational procedures", which leaves little room for the unique non-algorithmic reasoning that every person needs during encounters with healthcare systems. Therefore, our work focuses on understanding and reflecting on this nonalgorithmic reasoning within the domain of medicine. The design case reported in this paper is the MINI project (short for Mobile INteractlon), which explores the use of handheld computers for mobile e-learning (m-learning) in order to support junior registrar doctors at an emergency medical ward at a regional hospital in Denmark. Handheld computers or personal digital assistants (PDAs) have become common in clinical settings, and 

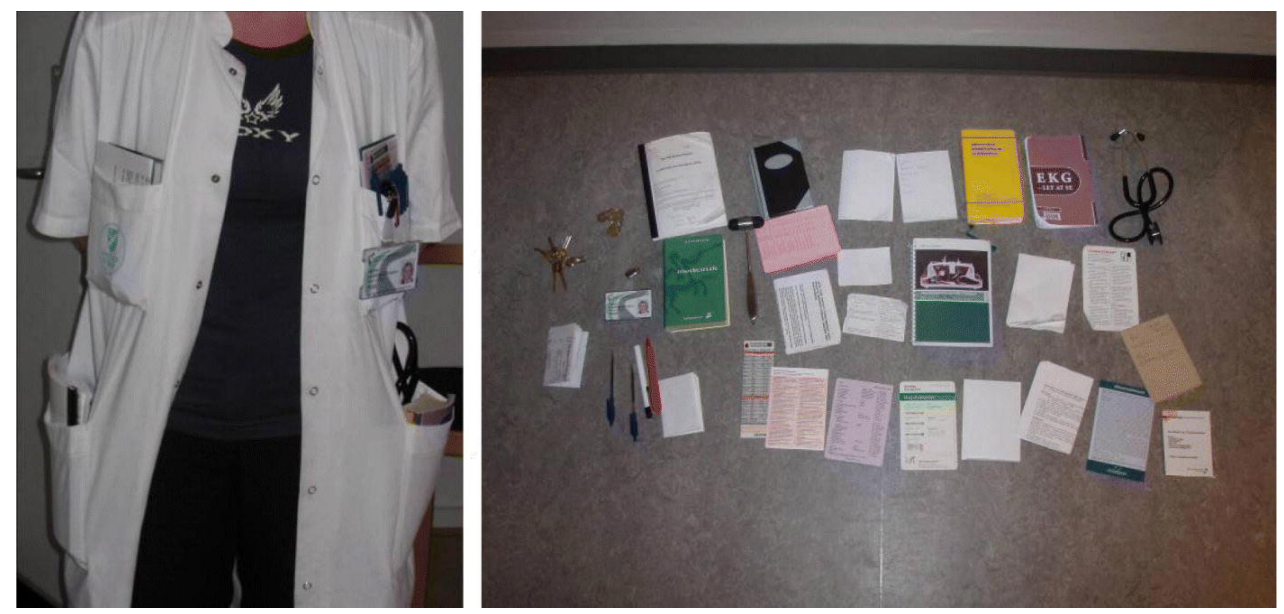

Figure 1.

Photograph 1: The white coat of a junior registrar doctor which illustrates the amount of information carried by a junior registrars $(2.6 \mathrm{~kg})$.

Photograph 2: The content of the pockets of that same white coat.

are used by physicians as well as nurses (Al- Ubaydli, 2004; Fisher et al., 2003). Generally, PDAs in clinical settings provide clinical reference guides for drug information, other clinical guidelines and patient tracking. Increasing wireless connectivity combined with more patient data in digital form introduces new application areas such as electronic prescribing, real-time medical records access, and point-of-care evidence-based literature searches (Rothschild et al., 2006). In other words, mobile technologies have reached a practical maturity level not only technically but also in relation to use in clinical settings. The MINI project builds on this maturity by developing a prototype for the design and use of m-learning resources for physicians, aimed at combining existing e-materials with new relevant functions, especially for junior registrar doctors. Our awareness of the theory/ practice dualism, in both software design and medicine, does not at first reveal a set of solutions for this design case, but rather a number of dilemmas. In random order and level of abstraction some of these dilemmas are:

- the already mentioned dilemma between the natural science theoretical foundation and the human science implementation of medical knowledge;

- the current "office-technology" framework of computer utilization compared with the nomadic clinical activity practiced and observed at the ward (Kanstrup \& Christiansen, 2006);

- the three-dimensional and visual nature of medicine (e.g. anatomy) and the twodimensional and text-based design of interfaces;
- the small screen of mobile technologies, which undermines the need to establish an overview of several complicated multiaxial parameters.

While this list is not exhaustive, it does present the most central dilemmas in the MINI project.

Design in this milieu of dilemmas yields a process of compromise between theory and practice, within both the computer-science and the medical domains. Moving toward a solution to this multifaceted design problem required that we focus mainly on abstract mental models utilized in order to operate within these many dilemmas, and how those mental models encouraged certain approaches to the development of the actual MINI application.

\section{POINT OF DEPARTURE: THE MINI PROJECT}

During their first years of medical practice, physicians need to operationalize the knowledge from medical studies, in the terms of Dreyfus and Dreyfus (1989). They move from knowing that to knowing how, in stages from novice to expert. Even as novices in a practical ward, junior registrar doctors often work alone with patients: analyzing results, diagnosing, and making initial care decisions. Learning support and back-up come in the form of pockets stuffed with reference books, look-up tables, instructions, and personal notes as shown in the photographs of a white coat with stuffed pockets, and the display of the contents of those same pockets (Figure 1).

The MINI project aims to experiment with the development of m-learning applications that support junior registrar doctors, especially in this first period of work. The hypothesis is that mobile information technology can provide not only 


\begin{tabular}{|c|c|c|}
\hline $\begin{array}{c}\text { PRACTICE TO } \\
\text { THEORY } \\
\text { How to understand } \\
\text { medical knowledge }\end{array}$ & $\leftrightarrow$ & $\begin{array}{c}\text { THEORY TO } \\
\text { PRACTICE } \\
\text { How to design for } \\
\text { medical knowledge }\end{array}$ \\
\hline
\end{tabular}

Figure 2.

The interaction between theory and practice in the MINIproject, which connects practice to theory by hypothesizing a way of understanding and supporting medical knowledge and then engages that hypothesis in the development of $\mathrm{m}$-learning applications within the specific context

lightweight learning support but also easy access to learning resources. That access is made possible by means of technological affordances like wi-fi, search functions, link structures, memory/space for personal notes, and the possibility of combining text with photographs, video, and sounds. In other words, mobile technologies have the potential to solve some of the dilemmas we identified, by introducing three-dimensional and visual information, breaking with the office metaphor, while focusing on mobility within a nomadic working environment (Bardram \& Bossen, 2005).

Within this problem space, we found it essential to do two things. First, we needed to focus on understanding and supporting the relationship between theory and practice within medical domains. Second, we needed to comprehend the move from theory to practice within the development of useful software that supports that domain. As shown in Figure 2 our work in these two areas led to a process where the initial point of departure connects practice to theory by hypothesizing a way of understanding and supporting medical knowledge, and then engaging that hypothesis in the development of m-learning applications within the specific context.

In the design process of the MINI project, we have tried to facilitate close and conscious interaction between theory and practice, with attention to results in product as well as process. We have used this focus on interaction to elucidate elements of the complex relationships within both medicine and the development of software. These complex relationships are accounted for through a series of domainspecific models, which are:
THE FOUR COMPONENTS

\author{
Knowledge \\ Teamwork \\ Technology utilization \\ Manual skills
}

Figure 3.

The four components in a model of a health care provision which are either latent, if appropriate, or active in every situation.

- a model of healthcare provision;

- a model of medical knowledge abstraction levels;

- a clinical workflow model for the initial care of a patient.

These models, along with the overarching heuristics they produced, account for how we both understand the domain and develop its tools.

In "New theoretical approaches for human computer interaction", Rogers (2004) calls for consideration of "the role theory should have in the field of HumanComputer Interaction (HCI) and the practice of interaction design" (p. 131). Rogers stresses that there is a need for theories that "best inform design" (p. 134) and that this need calls for focusing "more on the process of design" (p. 134), "on the nature of the relationship between researchers and designers" (p. 134), and on "building up a lingua franca - that different parties in research and design can use to point to common referents" (p. 134). The models presented in this paper point toward a common understanding not only of the myriad problems that interaction designers confront, but also, in this case, of our interaction with the physicians associated with the MINI project. A central point that emerges in these models, in relation to Rogers's perspective, concerns the lack of a one-to-one relationship between theory and practice. Consequently, the models are not theories simply informing practice. Rather these models describe processes, which function as artefacts in the MINI project, and also take on meaning as a lingua franca. 


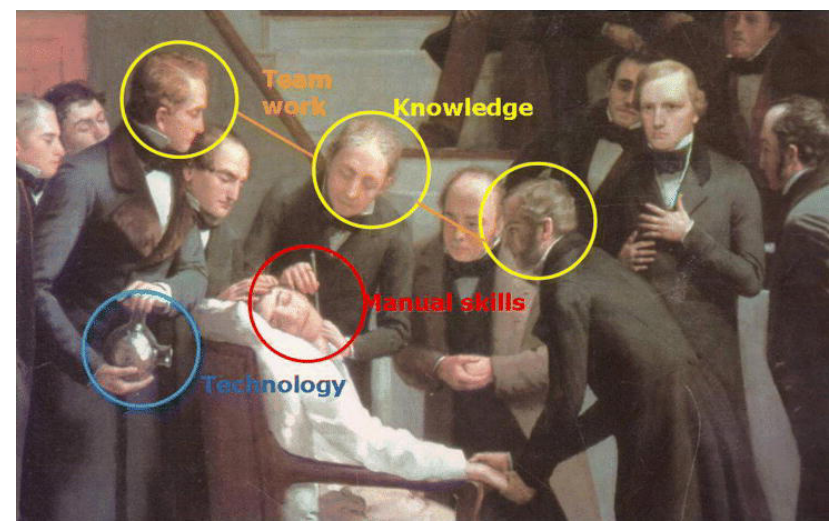

Figure 4.

An artistic representation of the first public demonstration of anaesthetics performed at Massachusetts General Hospital on 160 ctober 1846 with annotations by the authors showing the four components of health care provision. Detail from a painting by Robert Hinckley, The First Operation under Ether (1882).

Epistemologically, we refer to Dewey (1988/1929), who in "The quest for certainty" argues for the replacement of the old centre of the universe being "the mind knowing" by a new centre:

The new centre is indefinite interaction taking place within a course of nature which is not fixed and complete, but which is capable of direction to new and different results through the mediation of intentional operations. Neither self nor world, neither soul nor nature (in the sense of something isolated and finished in its isolation) is the centre, any more than either earth or sun is the absolute centre of a single universal and necessary frame of reference. There is a moving whole of interacting parts. (p. 232)

In general

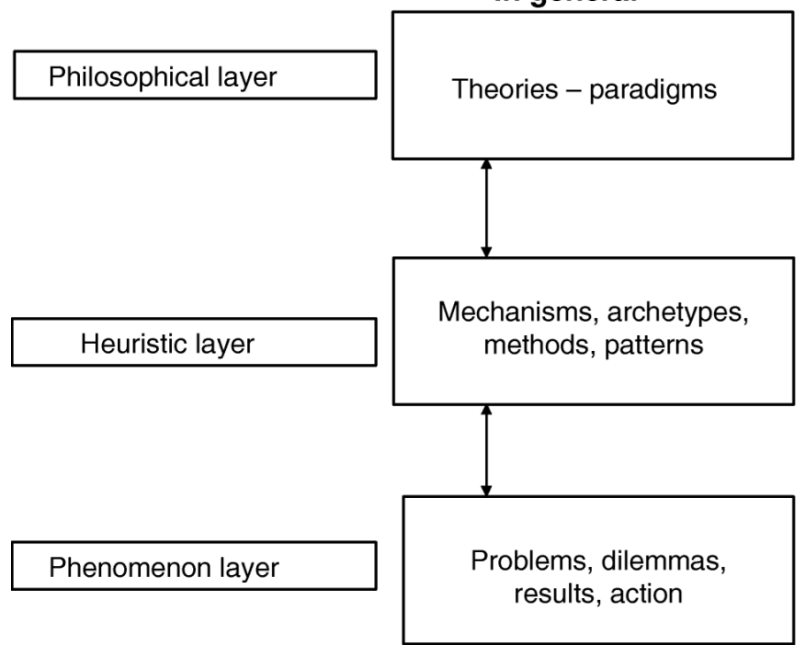

This "indefinite interaction" or "moving whole of interacting parts" is (of course at a more concrete level than presented by Dewey) the interaction presented in the models in this paper: non-algorithmic dynamics of medicine and design. The following presents this multifaceted interaction by first teasing apart the complexities of medicine from the complexities of developing an m-learning application that will be useful for practice. We then discuss the results that develop when theoretical models that interact with software development meet theoretical models that interact with medical practice.

\section{A THEORY OF MEDICAL KNOWLEDGE}

We present here a theory of medical knowledge as both a model of a healthcare provision, and a model of medical knowledge abstraction levels. The model of health care provision contains four general components (Figure 3). These are knowledge; teamwork; and technology utilization, such as the utilization of medications; and manual skills.

In self-care and non-patient conditions, the patient or a proxy can supply all four components. While all components need not be present in every provision, they should be latent for activation if appropriate. These components are illustrated by Robert Hinckley's painting of the first anaesthesia at Massachusetts General Hospital (Figure 4). Only the components "knowledge" and "team-work" can at the present stage of maturity in computer technology be sufficiently computersupported, making them part of the technology utilization component itself.

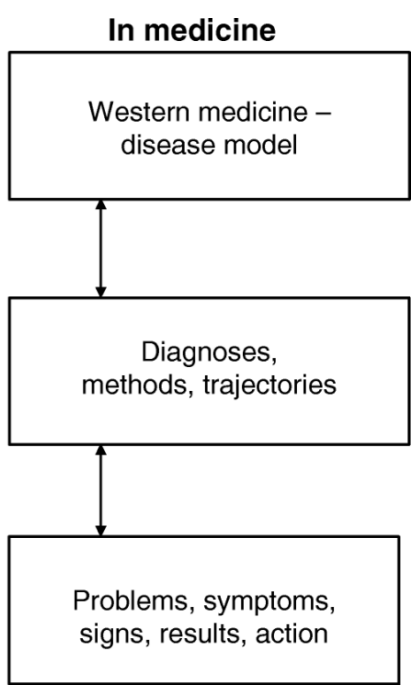

Figure 5 .

An employed model of knowledge on medicine. The western medicine model simply states: "That to every disease or syndrome is a specific lesion, this can be functional, anatomical, physiological, biochemical, genetic, or social" (interpretation of the authors). 


\section{THE MODEL OF KNOWLEDGE ABSTRACTION LEVELS IN MEDICINE}

Our model of knowledge in medical domains is inspired from Adolfsen's (1992) epistemological model of everyday problem-solving, where knowledge is stratified in three layers, which are the philosophical layer, the heuristic layer and the phenomenon layer. We define medical knowledge as consisting of those three interrelated layers (Figure 5).

The artwork in Figure 6 presents examples from the medical domain. These works visualize medical knowledge layers within that epistemological three-layer model. The first image illustrates the upper abstract layer, which contains knowledge about disease-causing mechanisms, specifically the tuberculosis bacteria symbolized by Pasteur in his laboratory (Edelfelt, 1885). The middle image illustrates an archetypical layer, which in this case shows the X-ray of a patient with tuberculosis. The third image illustrates the phenomenological layer, represented by Picasso's Science and Charity (1897), showing the individual patient, his problems, the manifestations of the disease, and actions by the doctor and relatives.

Importantly, a dilemma can coexist in three appearances of abstraction: at the bottom level, the concrete everyday problem is in contact with the everyday action and environment. In the middle heuristic layer, the general problem is an archetype, a pattern, a diagnosis, or a method. In the upper model layer, the overarching perspective of the problem emerges as a theory or philosophy. Put to a medical example, this issue can be described in the following way:

1. The phenomenon or patient's problem, which is palpitations, corresponds to the doctor's problem in this layer, which is tachycardia (fast heart rate).

2. The heuristic or archetypical layer in which the disease entities, such as thyrotoxicosis, fever, or heart disease are located relate to the problem of fast heart rate.

3. The theoretical or philosophical layer contains factors promoting a fast depolarization of the sinus node in the heart.
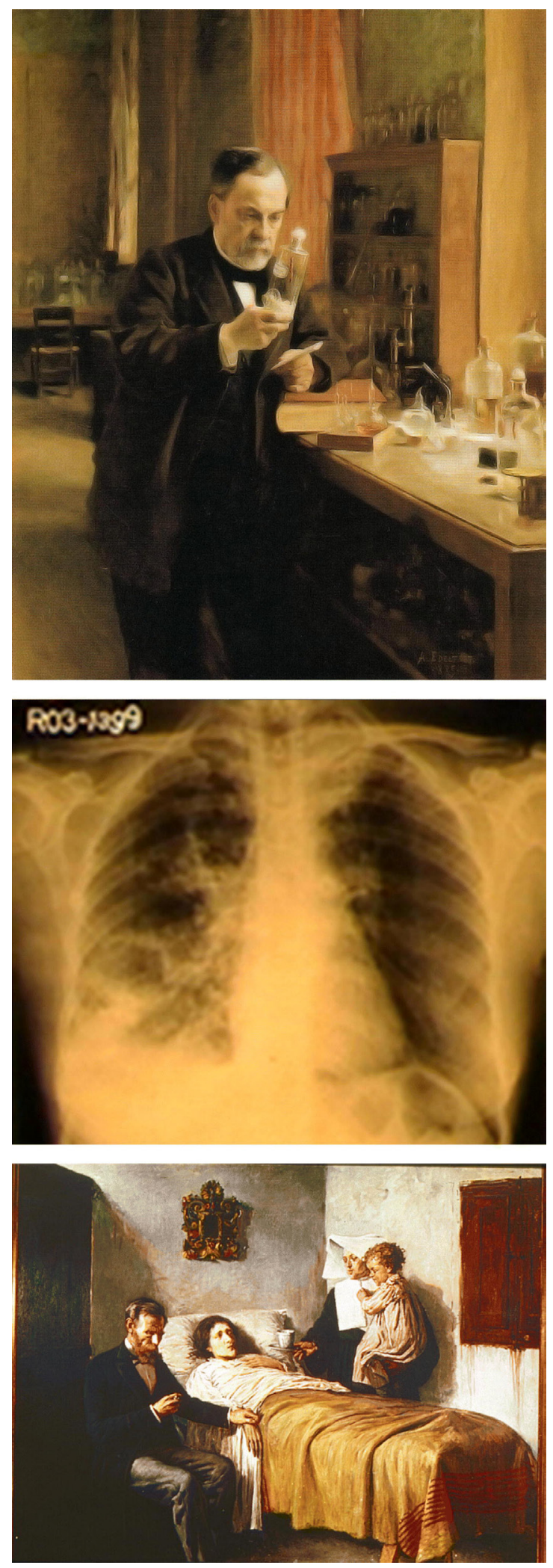

Figure 6 .

A visualization of the philosophical, heuristic, and phenomenon layers of medical knowledge. The painting in the upper panel is by Albert Edelfelt, Louis Pasteur in His Laboratory (1885).

The lower panel the phenomenon layer - is represented by a painting of the young Picasso (1897, now on show in Museum Picasso Barcelona (C) Succession Picasso/DACS 2007): Science and Charity. The heuristic layer is depicted by an X-ray picture of tuberculosis of the lung. 
Medical knowledge

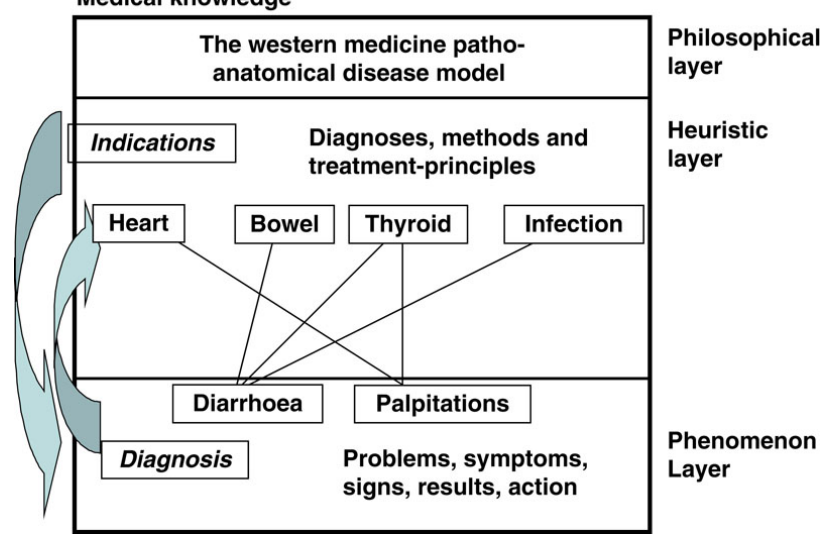

The example illustrates an instantiation of a problem that requires clinical reasoning. For that reasoning to emerge, the physician must engage in knowledge acquisition, translation of that knowledge, activation, and operationalization across different layers, which is not a straightforward algorithmic exercise.

A recent article, “Educational strategies to promote clinical diagnostic reasoning" (Bowen, 2006), discusses this process in detail, but mainly within the phenomenon layer.However, doctors need to master a problem in all three layers, including the action relevant to diagnostic and therapeutic procedures, as illustrated in Figure 7, which presents an example of the nonalgorithmic dynamics of the operationalization of medical knowledge. In it, a patient presents with diarrhoea and palpitations. The doctor generalizes these problems to a diagnosis. During this process he/she also activates knowledge from the upper theoretical layer. After the diagnostic process, an indication of treatment is formulated employing both theoretical knowledge and heuristic knowledge
Figure 7.

An example of the (non-algorithmic) dynamics of operationalization medical knowledge. A patient presents diarrhoea and palpitations. The doctor generalizes these problems to a diagnosis in the diagnostic process. During this process he activates knowledge from the upper theoretical layer. After the diagnostic process an indication of treatment is formulated employing both theoretical knowledge and heuristic knowledge about treatment principles. The principle is brought into action tailored to the specific problems and patient. The explanation for the choices made is the indication for the treatment.

about treatment principles. The principle is brought into action tailored to the specific problems of that specific patient. An explanation for the choices made produces the indication for the treatment.

\section{DESIGNING M-LEARNING FOR MEDICAL KNOWLEDGE}

Our layered, non-algorithmic perspective on medical knowledge calls for a focus on supporting a bridge among different layers. In the MINI project, we have worked with this challenge from the outset by considering the problem through the lens of archetypes. An archetype is a typical patient for the ward. At the emergency medical ward, examples of archetypes include feverish patients, rheumatic patients, stroke patients, heart arrhythmias, and suicidal behaviour. The focus on archetypes, as a point of departure for the miniapplication, is grounded in the heuristic layer. It assumes that the residents are respected professionals able to perform clinical reasoning. Clinical reasoning suggests that the resident can activate different layers of relevant
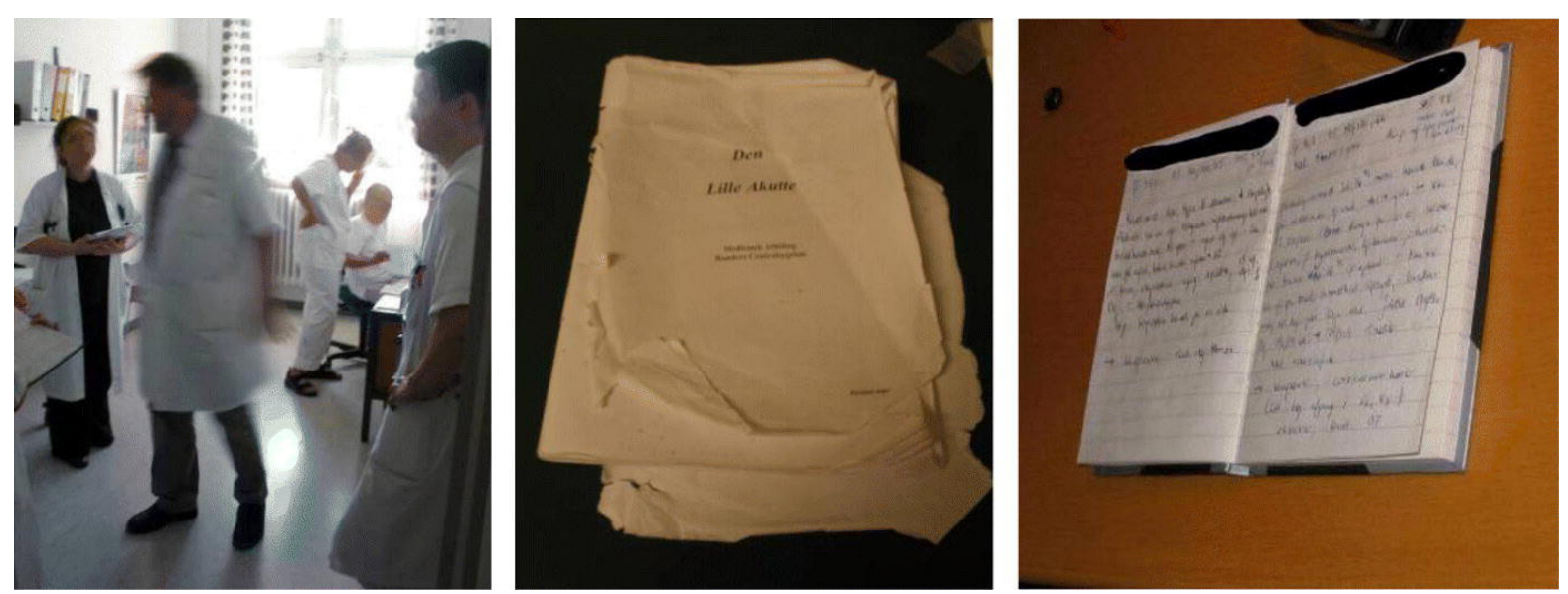

Figure 8.

The photograph on the left shows the most wanted but often most unavailable learning resource: the chief physician. Here he is seen giving advice briefly to registrars on duty while on his way to another task. The middle photograph shows the most used learn- ing resource: the ward instructions made by chief physicians at the ward. The photograph on the right shows a notebook used by most junior registrars to collect information during their work. In this way, they create their own personal archetypes. 


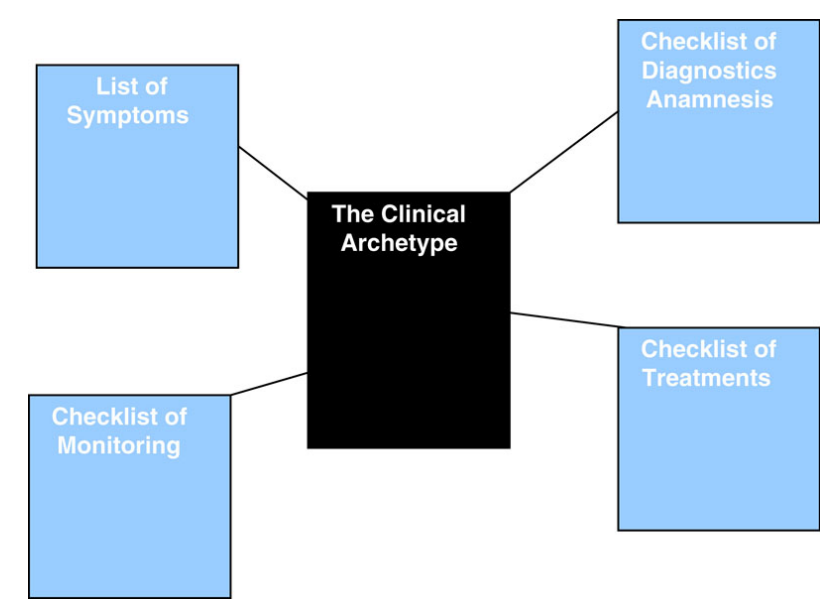

Figure 9 .

Four milestones that take on a structure as checklists, which are synthesized into clinical archetypes.

knowledge in the context of the specific patient - fitting her or him into the correct archetype, while performing the necessary patient-specific adjustments from a non-existing, average patient described in the archetype. This approach is utilized in order to reduce unwanted variations in healthcare provisions due to lack of knowledge while promoting individuality in patient care, which is not enhanced by "standard operational procedures" and "standard, algorithmic based patient models".

Our perspective on medical knowledge was established through observations, workshops, informal conversations, and theoretical discussions between interaction design researchers and physicians on the ward. In these situations, we found that archetypes served as the common foundation between these two different professions. Additionally, observations of physicians working on the ward provided preliminary insight for the designers concerning the situated meaning of mobility. While existing literature on m-learning seemed to define mobility as access to information at any time and any place (Metcalf, 2006), mobility on the medical ward means easy and quick access to information in the very specific sense of a maximum three-minute search. Consequently, the need for easy and quick interaction techniques, such as onehand interaction or voice interaction when on the move, along with the need to not only look up but also collect information in an easy and quick way, is critical. As noted by Anne Marie Kanstrup and Ellen Christiansen (2006), currently these interaction techniques take the form of personal notebooks (Figure 8).
Additionally, a workshop shed light on the junior registrars' favourite but often unavailable learning resource: chief physicians (Figure 8, left side); their most often used learning resources: ward instructions written by chief physicians (Figure 8, middle and right); and the many prioritized learning resources carried along in the pockets of the white coat (see Figure 1).

During this empirical research process, our emerging shared understanding concerning medical knowledge included the need to focus on:

- easy access with a three minute maximum search;

- ward instructions;

- the possibility of not only pulling but also putting information into an mlearning application for use among junior registrar doctors on the emergency medical ward.

On this basis, we started a process of digitalizing and minimizing existing ward instructions for PDAs in close cooperation with chief physicians on the ward. The designers transformed existing paper archetypes into digital archetypes on the basis of a modified general workflow model with four milestones. These milestones are situated in the phenomenon layer, and correspond with the conclusion of the diagnostic interview (anamnesis, symptoms and signs), which was often the basis for activating a specific archetype. The checklists for these milestones, which are illustrated in Figure 9 , include a checklist of:

- symptoms and signs containing specific ideas for further and alternative information relevant to a more precise positioning of the patient within the range of the archetypal diagnoses;

- diagnostics, objective findings, and tests containing relevant ideas for specific hospital procedures;

- treatment plans;

- monitoring and alternative actions. 


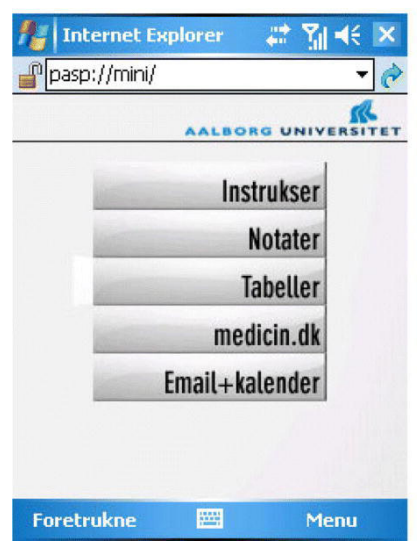

b

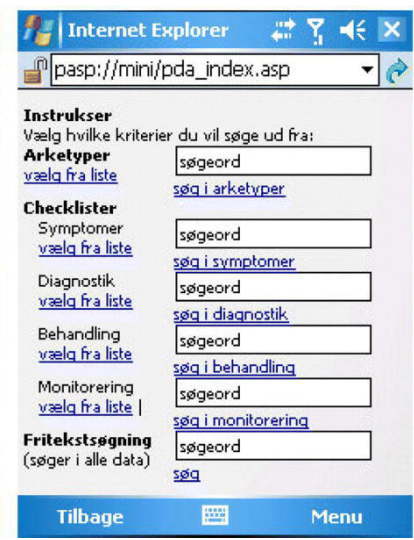

c

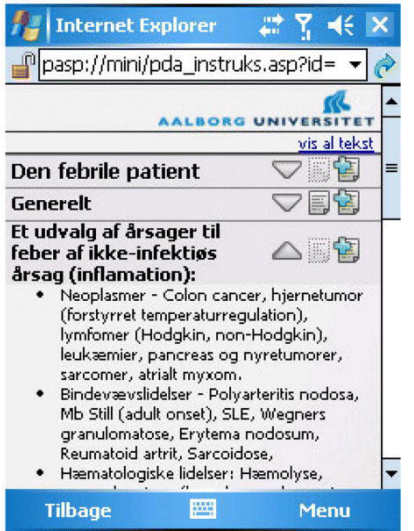

Figure 10.

Screen-dumps from the MINIapplication showing a) the front-page of the application, b) search functions and c) a minimized digital archetype organized in headlines which are unfolded/folded by use of the drop-down icon and linked to notes by use of the note/paper icon (being the icon for inserting notes and the clear/transparent icon displaying whether notes has been made).
The technical dimensions of these milestones indicated that we should transform the paper ward instructions by developing the following:

- an information architecture that would be useful for a database that incorporates the three layers;

- software that can act as an editor for producing archetypes within this structure;

- a navigation design to identify MINI archetypes within the environment of PDA interaction.

The software used as an editor for producing text for digital archetypes has been designed in Microsoft Word ${ }^{\mathrm{TM}}$. We used this application because the existing text (the existing paper instructions) was produced in Word. Additionally, this software is known to the archetype producers, in other words, the staff specialists and chief physicians who marked and prioritized the text for later hyperlinking by means of a parser constructed within the project.

The minimizing of the rather long text of each archetype (up to 25 regular pages) has been done by carefully developing the navigation for the text. In answer to the call for easy and quick interaction, we have focused on the following concerns. We considered not only the screen of the PDA but also the hardware as interface. For example, a shortcut to the MINI application has been programmed and works by using a button on the front of the PDA. We gathered central information in a MINI-front-page, which gives easy access to archetypes through a search function, allows for personal notes, permits the visualization of often used look-up tables (e.g. for medication, allergies, etc.) and links to the Danish website for information related to medical handbooks and common drug catalogues. A click on a logo of Aalborg University, present on every screen, takes the user back to the MINI front page (Figure 10a).

We not only provided direct access to specific information through search functions, giving options to conduct free text searches, but also supplied access to information organized within checklists (Figure 9) of symptoms, diagnostics, treatments, and monitoring (Figure 10b). We gave an overview of the archetypes (up to 25 regular Word pages) by providing headlines (designed in cooperation with the archetype writers) under which text can be unfolded by clicking the drop-down icon. And we delivered at-a-glance information in the archetypes that uncover annotations to the text, communicated through a transparent icon, when no notes are available, and a clear icon, when notes have been inserted. The addition of a " + " to the icon indicates readiness to add notes to the text (Figure 10c).

\section{DISCUSSION}

In the process of putting practice to theory and theory to practice the philosophical, heuristic, and phenomenon layers have functioned as a boundary object or a bridge between the physicians and interaction designers in the MINI project. These models of thought, which we have identified as layers of knowledge, not only worked as a shared language for understanding the practice domain (medical knowledge) but also provided a structure 
for the design of the MINI system's software and hardware. This paper has presented how such abstract models of thought can function as communication between the different partners in the design project, i.e. as an example of how we have worked on building up a "lingua franca" (Rogers, 2004, p. 134) in the MINI projects based on a shared frame of reference. Whether these models of thought are the best way to "inform design" as called for by Rogers (2004, p. 134) is, however, another question that we cannot answer in this paper. But we can say that the lack of a one-to-one relationship between theory and practice suggests that such a lingua franca must be mixed and elastic, giving room for a variety of words and phrases to support the "indefinite interaction" or "moving whole of interacting parts" (cf. Dewey 1988/1929, p. 232) of any design case.

\section{CONCLUSION}

Our work aims to describe models of thought, which can be visualized as philosophic, heuristic, and phenomenon layers, and utilized to operate between theory and practice in the design of an m-learning application for junior registrar doctors on a medical ward. The paper has presented in detail models for understanding medical knowledge, along with the results of the development of an m-learning application within this work domain. At a more general level, the paper has presented an epistemological perspective on the complexity faced within the domains of medicine and software design - a process where there is no one-to-one relationship between theory and practice, but an indefinite interaction between the two. Consequently, the discussion highlights the need for mixed and elastic perspectives on languages and methods for software design, which gives room for the indefinite interaction faced in every design project.

\section{ACKNOWLEDGEMENTS}

The authors wish to acknowledge the work of Pernille Bertelsen, Ellen Christiansen, Thomas Christiansen, and Christian Nøhr and to thank physicians on the medical ward for participating in observations, workshops, discussions, and pilot test. The MINI project was made possible due to grants from the Danish Ministry of Science and Technology.

\section{REFERENCES}

Adolfsen, J. (1992). Problemer i videnskab, en erkendelsesteoretisk begrundelse for problemorientering (Problems in science, an epistemological argumentation for problem-orientation). Aalborg, Denmark: Aalborg Universitetsforlag.

Al-Ubaydli, M. (2004). Handheld computers. British Medical Journal, 328, 1181-1184.

Bardram, J. E., \& Bossen, C. (2005). Mobility work: The spatial dimension of collaboration at a hospital. Computer Supported Cooperative Work, 14(2), 131-160.

Bowen, J. L. (2006). Educational strategies to promote clinical diagnostic reasoning. Medical Education, 355, 2217-2225.

Buchanan, R. (1996). Wicked problems in design thinking. In V. Margolin, \& R. Buchanan (Eds.), The idea of design (pp. 3-20). Cambridge, MA: MIT Press.

Dewey, J. (1988). The quest for certainty. In J. A. Boydston (Ed.), John Dewey the later works, 1925-1953: 1929/The quest for certainty Vol. 4 (p. 232). Carbondale: Southern Illinois University Press. (Original work published 1929).

Dreyfus, H. L., \& Dreyfus, S. (1989). Mind over machine: The power of human intuition and expertise in the era of the computer. Oxford, UK: Blackwell.

Fisher, S., Stewart, T. E., Mehta, S., Wax, R., \& Lapinsky, S. E. (2003). Handheld computing in medicine. Journal of the American Medical Informatics Association, 10(2), 139-149.

Floyd, C. (Ed.). (1992). Software development and reality construction. Berlin, Germany: Springer-Verlag.

Kanstrup, A. M. \& Christiansen, E. (2006). Mobility at a medical ward. In 0. W. Bertelsen et al. (Eds.), Proceedings from the 2006 DHRS/ Danish HCI Research Symposium, Aarhus, Denmark, DAIMI PB - 580, 31-32.

Mathiassen, L., \& Stage, J. (1992). The principle of limited reduction in software design. Information Technology \& People, 6(23), 171-185.

Metcalf, D. S. (2006). mLearning: Mobile learning and performance in the palm of your hand. Amherst, MA: HRD Press.

Rogers, Y. (2004). New theoretical approaches for humancomputer interaction. Annual Review of Information, Science and Technology, 38, 87-143.

Rothschild, J. M., Fang, E., Liu, V., Litvak, I., Yoon, C., \& Bates, D. W. (2006). Use and perceived benefits of handheld computer-based clinical references. Journal of the American Medical Informatics Association, 13(6), 619-626.

\section{CORRESPONDENCE}

Anne Marie Kanstrup,

Department of Communications, Kroghstraede 1, DK-9220 Aalborg, Denmark.

E-mail: kanstrup@hum.aau.dk

ISSN 1749-3463 print/ ISSN 1749-3471

DOI: $10.1080 / 17493460701800231$

(C) 2007 Danish Centre for Design Research 